\title{
Experimental study on SAR reduction from cell phones
}

\author{
Marius-Vasile Ursachianu ${ }^{1}$, Ovidiu Bejenaru ${ }^{1}$, Catalin Lazarescu ${ }^{1}$, Alexandru Salceanu ${ }^{2}$ \\ ${ }^{1}$ Romanian "National Authority for Management and Regulation in Communications" (ANCOM), Romania \\ 2 "Gheorghe Asachi" Technical University of Iasi, Romania
}

\section{ABSTRACT}

The actual problem of the human exposure to different types of electromagnetic field sources is a challenging one and should be considered an up to date issue due to one major actual trend: the increasingly intensive penetration of various wireless communication technologies in virtually all places and times that make up our daily lives. The here presented paper presents an experimental study focused on measurements for three types of mobile phones, belonging to different generations, operating at the two characteristic frequencies of the GSM bandwidth. We have been used a SATIMO-COMOSAR evaluation dosimetry system, provided by the LICETER laboratory of ANCOM Romania. The determined values of the absorbed incident energy by the tissue of a mannequin (phantom) model for the human head, which is part of the dosimetry system, have been also compared with those obtained in the case when the mobile phones are protected by multilayer cases, aiming to study their possible limiting effect. The influence that "touch" or "tilt" positions might have on absorbed (by the human head model) incident energy values has also been investigated. The comparative processing of the obtained results allowed the formulation of recommendations on reducing the exposure to electromagnetic radiation associated with the use of the mobile phone. This paper is an extended and improved version of the original contribution to the IMEKO TC 42020 virtual conference.

\section{Section: RESEARCH PAPER}

Keywords: Absorbed incident energy; Human exposure measurement; Near field exposure; CST simulation; Electromagnetic field dosimetry; SR EN 62209-1

Citation:Marius-Vasile Ursachianu, Ovidiu Bejenaru, Catalin Lazarescu, Alexandru Salceanu, Experimental study on SAR reduction from cell phones, Acta IMEKO, vol. 10, no. 2, article 21, June 2021, identifier: IMEKO-ACTA-10 (2021)-02-21

Section Editor: Ciro Spataro, University of Palermo, Italy

Received January 24, 2021; In final form April 29, 2021; Published June 2021

Copyright: This is an open-access article distributed under the terms of the Creative Commons Attribution 3.0 License, which permits unrestricted use, distribution, and reproduction in any medium, provided the original author and source are credited.

Corresponding author: Alexandru Salceanu, e-mail: asalcean@tuiasi.ro

\section{INTRODUCTION}

The possible effects of ambient electromagnetic fields on human beings are general sources of concern and legitimate questions. For this reason, scientific research in the field has been strongly supported by diverse bodies and organizations, national and international, being materialized through the adoption of recommendations, setting limits and developing guidelines. This multitude and diversity provided results which, although fundamentally convergent in nature, were quite different in form. This is the main motivation for the current trend towards harmonization, providing benefits for authorities, industry and clients. Essentially, the study on human exposure has two important parts: first setting limits and second establishing the correct measurement to verify compliance with previously accepted limits. For the first part, based on extremely in-depth interdisciplinary research, most countries around the world have given a positive response to the rational scientific underpinning the ICNIRP limits. These reference levels have also been assumed by the International Telecommunication Union (the
United Nations specialized agency) and the World Health Organization. These limits are approximately in compliance with the North American FCC Guidelines developed by the Federal Communications Commission, Office of Engineering \& Technology. In terms of the measurement techniques and practice there is even greater diversity. For example, the assessment of specific absorption rate of human exposure to radio frequency fields from hand-held Wireless Communications Devices is an important concern of the profile organizations, especially since the approaches are in a permanent dynamic. For example, the well-known IEEE Standard 1528, first issued in 2003, has been significantly updated and completed in 2013 and 2020, respectively. Its quasi-universal character has been enhanced through the adoption by the International Electrotechnical Commission of the two measurement standards in place IEC 62209 (part 1, also here applied and part 2). As concerning the penetration of electromagnetic radiations in the human body for different radio frequencies, ICNIRP limits for the specific absorption rate (SAR) have been assumed: $0.08 \mathrm{~W} / \mathrm{kg}$ on average for the whole human body, respectively 
$2 \mathrm{~W} / \mathrm{kg}$ for SAR located in the head or trunk area (general public exposure). These SAR values are averaged over 6 minute exposure time and $10 \mathrm{~g}$ of tissue. [1], [2], [3], [4].

The main objective of this paper is to determine and to compare the SAR values, for three different generation mobile phones, using a SATIMO-COMOSAR system and considering different exposure scenarios. Two of these phones are old models, being released on market in 2009 and respectively in 2012. The third is a newer model, released on market in 2018. We have been interested to observe if the generation of the mobile phones (design, position of antenna, housing materials) has a significant impact over SAR values (determined for different positions of mobile phone related to the head phantom, SAM). The specific anthropomorphic mannequin (SAM) has been designed to provide a conservative estimation of the actual peak spatial specific absorption rate (SAR) of the electromagnetic field radiated by mobile phones, [5].Complementary, for the new smartphone released in 2018, the effect that "touch" or "tilt" positions might have on SAR values has also been studied. The determined SAR values have been also compared with those obtained in the case when the mobile phones are protected by multilayer cases, aiming to study these cases possible limiting impact.

The shape of the SAM physical model has been derived in a percentage of $90 \%$ from the shape of an adult male head; its dimensions have been reported in [6]. The shape of the ears has been adapted to represent the flattened ones of a phone user.

Various studies propose different kind of shields for mobile phones to reduce the amount of power absorbed in the head, aiming to minimize the health effects [7], [8].

Moreover, researches focused on SAR reduction due to the type of antennas (PIFA or Helical), placed at the top or at the bottom of the device, have been also carried out [9], [10], [11].

Finally, the SAR values directly determined by us using the calibrated SATIMO-COMOSAR dosimetry system for different exposure scenarios have been compared with each other and related to the limits accepted by the standards.

Ourstudies here presented could lead to the development(also including the direct involvement of human health specialists and bodies) to a series of recommendations and informal guidelines for effective reduction of human exposure to electromagnetic fields generated by wireless communications systems.

\section{MATERIAL AND METHODS}

The dosimetric quantity used for the evaluation of absorbed incident energy by the tissue is the Specific Absorption Rate (SAR). This parameter has been introduced for measuring the rate of energy absorbed by human body when it is exposed to a radio frequency electromagnetic field, the formula being given by the equation (1):

$$
S A R_{l}=\frac{\sigma}{2 \rho_{m}}\left|\vec{E}_{i}\right|^{2}=\frac{\omega \varepsilon_{0} \varepsilon_{r}^{\prime \prime}}{2 \rho_{m}}\left|\vec{E}_{i}\right|^{2},
$$

where $\varrho_{m}$ is the material density (in an elementary volume), $\sigma$ is the electric conductivity, $\varepsilon_{r}{ }^{\prime \prime}$ represents relative electric permittivity as a frequency depending function while $\mathrm{SAR}_{1}$ represents a local quantity, [12].

\subsection{The SATIMO-COMOSAR system for SAR evaluation}

The dosimetry evaluation system used for our measurements is competent to determine the distribution of SAR inside a human head phantom, so called SAM (Specific
Anthropomorphic Mannequin). The used phantom [13], is in accordance with American and European standards [14], [15].

Dosimetry assessment could be done both for the situations when the mobile phone is positioned by the right or by the left ear.

The main components of the used system, Figure 1, are: Kuka KR5 robot with his specific controller Kuka KRC2sr, [16] with electric field probe (calibrated before the measurement process), the SAM twin phantom, the liquids that simulate the human tissue for specific frequencies, a clamping device for the mobile phone under test, a signal generator Rohde \& Schwarz CMU 200 (a GSM base simulator that can control the output power and frequency) and a desktop PC running OpenSAR software, [17].

SAM phantom structure is made out from low loss and low permittivity material, embedded in wood.

The electric field (immersive probe inside the liquid simulating the dielectric properties of the head for different frequencies) is a triple dipole type (model EP96 - SATIMO). Efield probe provides an omni-directional response.

The clamping device for holding the mobile phone is also made from a low permittivity and low losses material, not to have any influence over the measured SAR values. The clamping device can be moved along three orthogonal axes $\mathrm{Ox}, \mathrm{Oy}, \mathrm{Oz}$ and it can be rotated around the phantom ear for precise positioning of the phone.

The OpenSAR software controls all robot movements, it determines local SAR values; as post processing application, it calculates SAR values, averaged on $10 \mathrm{~g}$ or $1 \mathrm{~g}$ of tissue.

\subsection{The SAR measuring procedure}

All the phases describing the SAR measurement procedure are widely depicted in SR EN 62209-1: 2007 [18].

The SAR evaluation for different frequencies has been done for each radio channel: low, middle and respectively high.

Two positions (illustrated in Figure 2) have been considered: the normal position (when the phone is on the cheek plane, also called "touch" or "cheek" position) and the tilt position (when

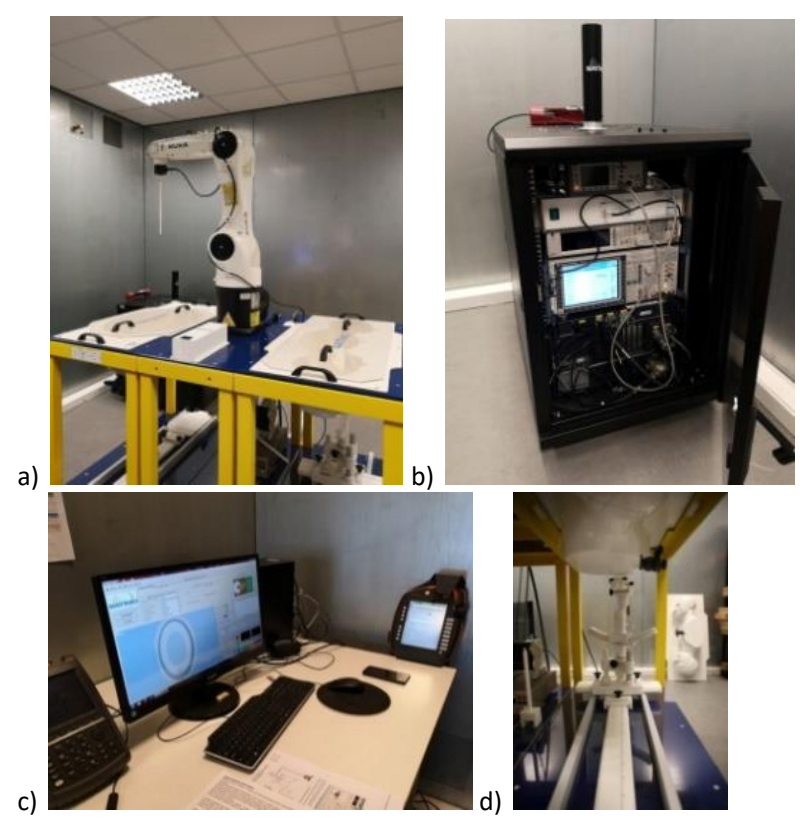

Figure 1. The SATIMO-COMOSAR system used for SAR measurements: (a) COMOSAR test bench andKUKA robot, (b) signal generator Rohde \& Schwarz CMU 200, (c) computing unit with OPENSAR software installed for testing system control (d) the fastening system used to secure the measuring equipment. 


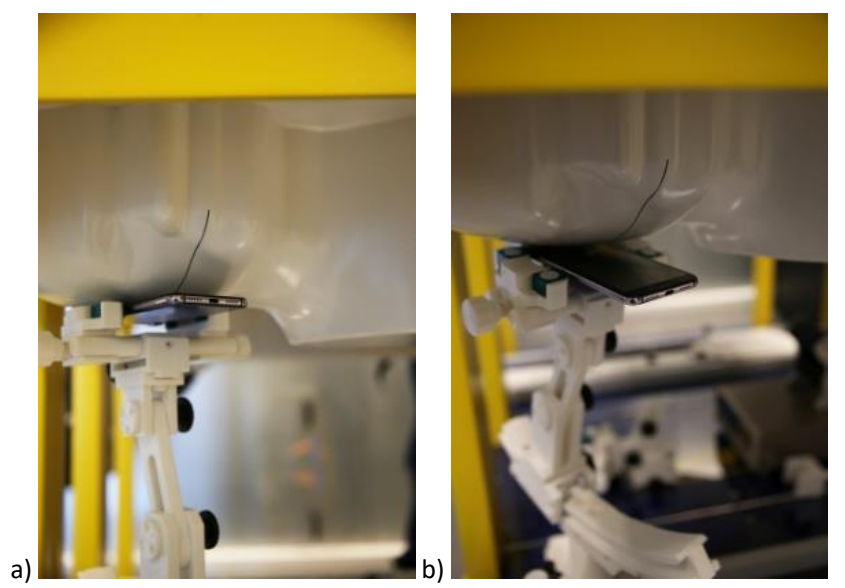

Figure 2. The cheek (a) and the tilt (b) positions of the Huawei P20 Pro mobile phone on the right side (ear) of SAM phantom.

the phone is tilted by 15 degrees toward cheek plane). Only one measurement location (of the SAM phantom) has been selected: the right ear.

The SAR evaluation has been done for two frequencies (GSM-900 and GSM-1800 bandwidth), $897 \mathrm{MHz}$ and $1747 \mathrm{MHz}$, respectively.

The mobile phone has been used with its internal transmitter, antenna, battery and all accessories supplied by the manufacturer. It is important as the battery to be fully charged before each test, for every case of exposure scenario taken into consideration.

Complementary, in Figure 3 is presented the front and the back side of Huawei P20 Pro mobile phone, inserted in a multilayer protective case made from hard plastic material.

For every position of the mobile phone tested for SAR evaluation, the following conditions should be fulfilled:

- existence of permanent radio connection between the base station simulator and mobile phone device at maximum power;

- SAR measurement should be done in a network of equally spaced points on a surface located at a constant distance from the inner surface of the phantom;

- SAR measurement should be done in equidistant points, in a cube located around the place where the maximum value of the field has been determined (by the probe who scan inside the phantom);

- calculation of the measured SAR as average value for $1 \mathrm{~g}$ and $10 \mathrm{~g}$
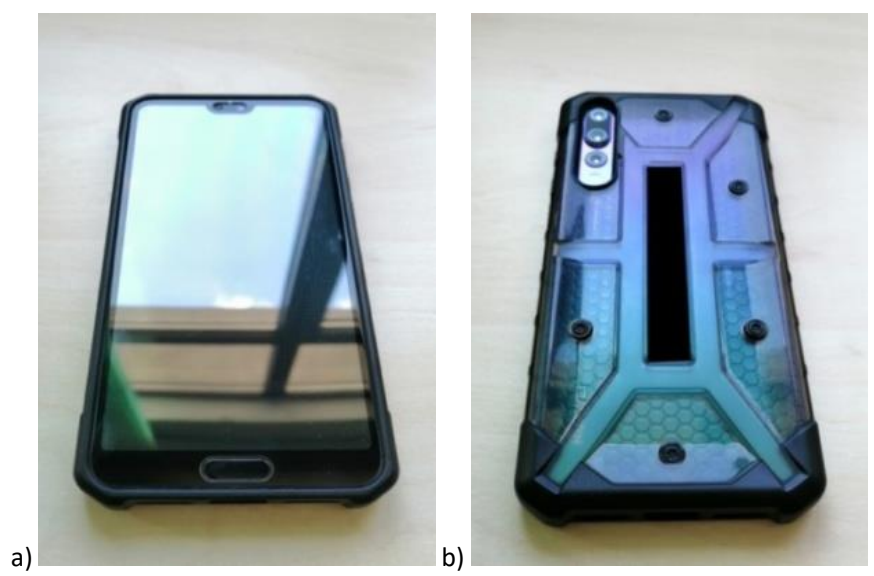

Figure 3. The front side (a) and the back side (b) of the Huawei P20 Pro mobile protected by a multilayered protective case.
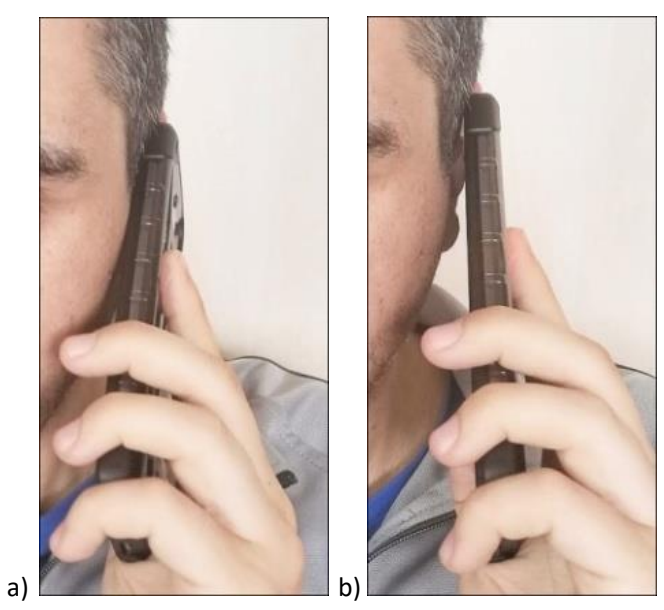

Figure 4. The Huawei P20 Pro phone with a protective case: cheek (a) and tilt (b) position, the two most common positions to the user's cheek.

- any others perturbation sources must be avoided inside the test room or in immediate vicinity.

Figure 4 shows a human user holding the Huawei P20 Pro mobile phone with the protective case in the cheek (touch) position, respectively the tilt position, the two selected situations of exposure.

\section{RESULTS AND DISCUSSIONS}

Operating procedure used during these measurements: a GSM communication has been established between the mobile phone under test and the base station simulator CMU200 for measuring the specific absorption rate (SAR).

The GSM 900 experimental conditions (for cheek or tilt positions, right or left) include: phantom - right head and left head; signal - TDMA (crest factor: 8.0); channel - middle; frequency $-897.59 \mathrm{MHz}$ (uplink); relative permittivity (real part) - 41.5; relative permittivity (imaginary part) -19.4 ; conductivity $(\mathrm{S} / \mathrm{m})-0.967$.

The GSM 1800 experimental conditions(for cheek or tilt positions, right or left) include: phantom - right head and left head; signal - TDMA (crest factor: 8.0); channel - middle; frequency $-1747.4 \mathrm{MHz}$ (uplink); relative permittivity (real part) - 40.102; relative permittivity (imaginary part) - 14.096; conductivity $(\mathrm{S} / \mathrm{m})-1.368$.

The SAR values for the HUAWEI P20 Pro mobile phone subject to dosimetric evaluation are shown in Table 1 for different positions (left and right side of the SAM phantom).

The values of the SAR for the SAMSUNG GT-S6102 mobile phone subjected to dosimetric evaluation are shown in Table 2 for the same placement of the phone: left and right side of the SAM phantom.

The corresponding SAR values for the NOKIA 2330c-2 mobile phone are synthesized in Table 3 .

A comparison of the SAR values when HUAWEI P20 Pro, SAMSUNG GT-S6102, NOKIA 2330c-2 mobile phones are positioned on the right side of the SAM phantom is presented in Table 4 (the test frequency in the GSM-900 band).

This comparison has been performed aiming to see if the SAR values averaged over $10 \mathrm{~g}$ of tissue for Samsung and Nokia phones are higher than the corresponding value for the Huawei phone.

The maximum value of SAR $10 \mathrm{~g}$ for Huawei, Samsung and Nokia mobile phones investigated in this evaluation dosimetry study is represented by OpenSAR software in $2 \mathrm{D}$ graphical 
Table 1. SAR values for different positionsof Huawei P20 PRO mobile phone relativeto the SAM phantom, two frequencies (GSM-900 and GSM-1800 bandwidth).

\begin{tabular}{cccccc}
\hline Bandwidth & Channel & Position & $\begin{array}{c}\text { SAR Peak } \\
\text { W/kg }\end{array}$ & $\begin{array}{c}\text { SAR 10g } \\
\text { W/kg }\end{array}$ & $\begin{array}{c}\text { SAR 1g } \\
\text { W/kg }\end{array}$ \\
\hline GSM 900 & Middle & Right - Cheek & 0.57 & 0.2854 & 0.4149 \\
GSM 900 & Middle & Right - Tilt & 0.28 & 0.1375 & 0.1945 \\
GSM 900 & Middle & Left - Cheek & 0.36 & 0.1735 & 0.2457 \\
GSM 900 & Middle & Left - Tilt & 0.16 & 0.0877 & 0.1196 \\
GSM 1800 & Middle & Right - Cheek & 0.34 & 0.1365 & 0.2282 \\
GSM 1800 & Middle & Right - Tilt & 0.14 & 0.0422 & 0.0713 \\
GSM 1800 & Middle & Left - Cheek & 0.15 & 0.0594 & 0.0974 \\
GSM 1800 & Middle & Left - Tilt & 0.05 & 0.0244 & 0.0358 \\
\hline
\end{tabular}

Table 2. SAR values for different positionsof SAMSUNG GT-S6102 mobile phone relative to the SAM phantom, two frequencies (GSM-900 and GSM1800 bandwidth).

\begin{tabular}{cccccc}
\hline Bandwidth & Channel & Position & $\begin{array}{c}\text { SAR Peak } \\
\text { W/kg }\end{array}$ & $\begin{array}{c}\text { SAR 10g } \\
\text { W/kg }\end{array}$ & $\begin{array}{c}\text { SAR 1g } \\
\text { W/kg }\end{array}$ \\
\hline GSM 900 & Middle & Right - Cheek & 2.31 & 0.7514 & 1.4469 \\
GSM 900 & Middle & Right - Tilt & 1.01 & 0.4936 & 0.7204 \\
GSM 1800 & Middle & Right - Cheek & 1.40 & 0.5081 & 0.9121 \\
GSM 1800 & Middle & Right - Tilt & 0.61 & 0.2268 & 0.3898 \\
GSM 1800 & Middle & Left - Cheek & 1.14 & 0.4909 & 0.8165 \\
GSM 1800 & Middle & Left - Tilt & 0.45 & 0.1686 & 0.2942 \\
\hline
\end{tabular}

Table 3. SAR values for different positionsof NOKIA 2330c-2 mobile phone relative to the SAM phantom, two frequencies (GSM-900 and GSM-1800 bandwidth).

\begin{tabular}{cccccc}
\hline Bandwidth & Channel & Position & $\begin{array}{c}\text { SAR Peak } \\
\text { W/kg }\end{array}$ & $\begin{array}{c}\text { SAR 10g } \\
\text { W/kg }\end{array}$ & $\begin{array}{c}\text { SAR 1g } \\
\text { W/kg }\end{array}$ \\
\hline GSM 900 & Middle & Right - Cheek & 1.92 & 0.8813 & 1.3496 \\
GSM 900 & Middle & Right - Tilt & 1.13 & 0.5301 & 0.7887 \\
GSM 1800 & Middle & Right - Cheek & 1.89 & 0.5611 & 1.0970 \\
GSM 1800 & Middle & Right - Tilt & 0.37 & 0.1386 & 0.2466 \\
GSM 1800 & Middle & Left - Cheek & 1.36 & 0.4709 & 0.8726 \\
GSM 1800 & Middle & Left - Tilt & 0.76 & 0.2356 & 0.4791 \\
GSM 1800 & Middle & Left - Tilt & 0.32 & 0.1056 & 0.1799 \\
\hline
\end{tabular}

Table 4. SAR Max (10g) values for Huawei vs Samsung and vs Nokia mobile phones, bandwidth: GSM-900, positions: right - cheek and right - tilt.

\begin{tabular}{ccccccc}
\hline Phone & $\begin{array}{c}\text { SAR 10g } \\
\text { (Right- } \\
\text { Cheek) } \\
\text { W/kg }\end{array}$ & $\begin{array}{c}\text { SAR 10g } \\
\text { (Right- } \\
\text { Tilt) } \\
\text { W/kg }\end{array}$ & $\begin{array}{c}\text { Samsung } \\
\text { vs Huawei } \\
\text { (Right- } \\
\text { Cheek) }\end{array}$ & $\begin{array}{c}\text { Samsung } \\
\text { vs } \\
\text { Huawei } \\
\text { (Right- } \\
\text { Tilt) }\end{array}$ & $\begin{array}{c}\text { Nokia } \\
\text { vs } \\
\text { Huawei } \\
\text { (Right- } \\
\text { Cheek) }\end{array}$ & $\begin{array}{c}\text { Nokia } \\
\text { vs } \\
\text { Huawei } \\
\text { (Right- } \\
\text { Tilt) }\end{array}$ \\
\hline Huawei & 0.2854 & 0.1375 & - & - & - & - \\
Samsung & 0.7514 & 0.4936 & 2.63 & 3.58 & - & - \\
Nokia & 0.8813 & 0.5301 & - & - & 3.08 & 3.85 \\
\hline
\end{tabular}

representation as a rectangular surface for the right or for the left part of SAM phantom face, according to the place where the phone have been placed for SAR evaluation. Around the position where the maximum SAR has been located, position marked with the red color, the software draws a rectangular surface. In Figure 5 is represented the surface SAR for Huawei, Samsung and Nokia mobile phones on the Left - Cheek position, $897.59 \mathrm{MHz}$, GSM-900 frequency.
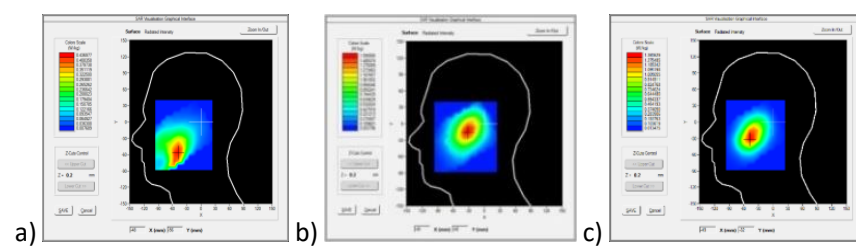

Figure 5. Surface SAR for Huawei (a), Samsung (b), Nokia (c) mobile phones for $897.59 \mathrm{MHz}$ on Left - Cheek.
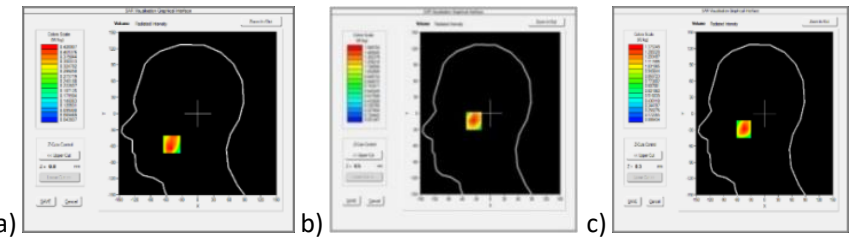

Figure 6. Volume SAR for Huawei (a), Samsung (b), Nokia (c) mobile phones for $897.59 \mathrm{MHz}$, Left - Cheek.

The OpenSAR software also can associate the surface where the maximum value of SAR $10 \mathrm{~g}$ have been found to a specific volume concentrated around this maximum value of SAR determined by probe during the scan process inside SAM phantom. In Figure 6 is represented the volume SAR for Huawei, Samsung and Nokia mobile phones on the Left - Cheek position, GSM-900 frequency band.

The SAR values for the HUAWEI P20 Pro mobile phone protected by a multilayer plastic case are shown in Table 5 for the right side of the SAM phantom, for both frequencies.

The comparative graphical distribution of SAR values (averaged on $10 \mathrm{~g}$ tissue) following the dosimetric evaluation of the Huawei P20 Pro (with and without protective case) is shown

Table 5. SAR values for different positions of Huawei P20 PRO phone with the multilayer protective case relative to the right part of SAM Phantom

\begin{tabular}{ccccc}
\hline Bandwidth & Channel & Position & $\begin{array}{c}\text { SAR 10g } \\
\text { W/kg }\end{array}$ & $\begin{array}{c}\text { SAR1g } \\
\text { W/kg }\end{array}$ \\
\hline GSM 900 & Middle & Right-Cheek & 0.1007 & 0.1525 \\
GSM 900 & Middle & Right-Tilt & 0.0323 & 0.0443 \\
GSM 1800 & Middle & Right-Cheek & 0.0348 & 0.0574 \\
GSM 1800 & Middle & Right-Tilt & 0.0140 & 0.0235
\end{tabular}

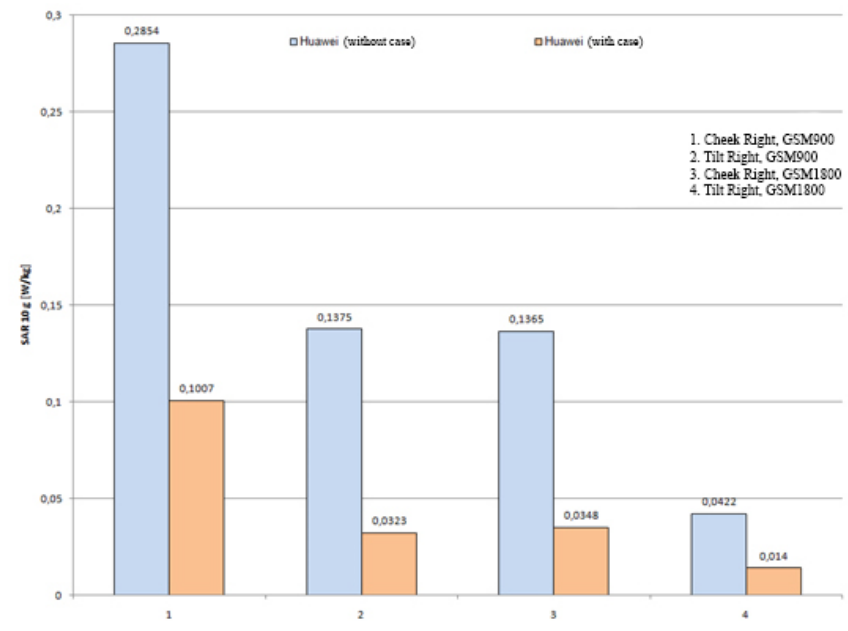

Figure 7. Comparative graphical distribution of SAR values ( $10 \mathrm{~g}$ of tissue) for the dosimetric evaluation of the Huawei P20 Pro: with and without multilayer protective case 
Table 6. Comparisons between SAR values (10 g) given by dosimetric evaluation of HUAWEI P20 PRO (provided or not with a protective case)

\begin{tabular}{ccccc}
\hline Bandwidth & Position & $\begin{array}{c}\text { SAR 10g in W/kg } \\
\text { Huawei } \\
\text { (no case) }\end{array}$ & $\begin{array}{c}\text { Huawei } \\
\text { (with case) }\end{array}$ & $\begin{array}{c}\text { Huawei } \\
\text { (no case vs }\end{array}$ \\
\hline wSM case)
\end{tabular}

in Figure 7. Four situations have been considered: cheek and tilt positions, for both frequencies of interest.

The SAR values recorded by the dosimetric evaluation of the Huawei P20 Pro (with and without case) are synthesized in Table 6 (for cheek and respectively tilt positions).

Figure 8 represents the surface SAR for Huawei P20 Pro phone, without case, on the Left - Cheek position for 897.59 $\mathrm{MHz}$ frequency.

The OpenSAR software can also associate the surface where the maximum value of SAR $10 \mathrm{~g}$ have been found to a specific volume concentrated around this maximum value of SAR determined during the scan process inside SAM phantom.

Figure 9 represents the volume SAR for Huawei, P20 Pro phones without multilayer protective case on the Left - Cheek position, GSM-900 frequency band.

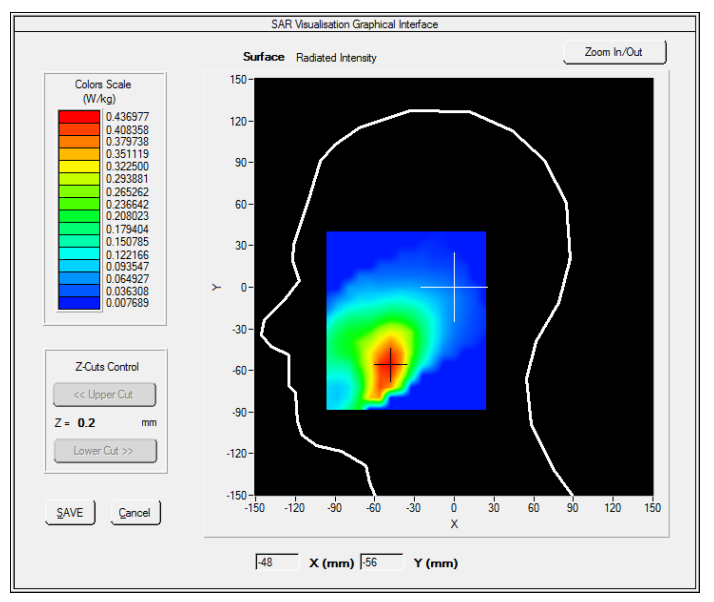

Figure 8. Surface SAR, Huawei P20 Pro phone without protective multilayer case.

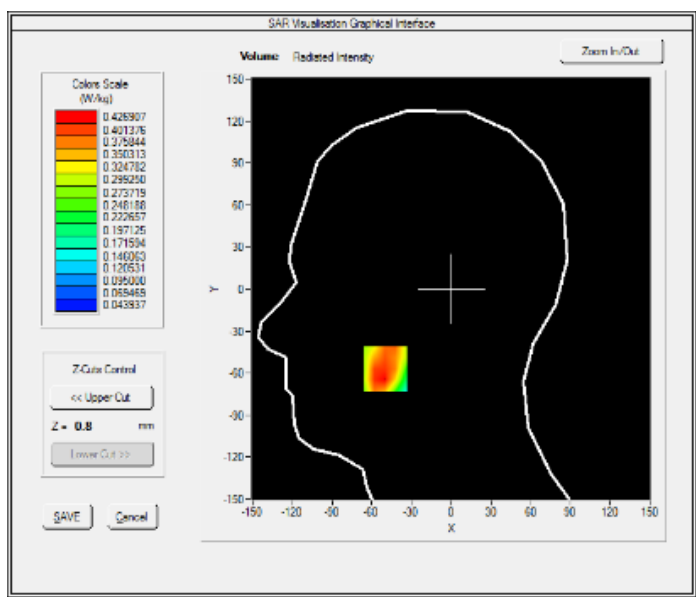

Figure 9. Volume SAR for Huawei P20 Pro mobile phone without protective multilayer case.

\section{CONCLUSIONS}

This paper present a set of SAR measurements performed for three, different generations, mobile phone devices: HUAWEI P20 Pro, SAMSUNG GT-S6102 and NOKIA 2330c-2, respectively. There is an over decade difference in term of market release between these phone models. The Huawei P20 Pro mobile phone is the newest one, being released on the market in 2018, while the two other mobile phones have been released on market in 2012 (Samsung) and even in 2009 (Nokia). We have also determined the SAR values when Huawei mobile phone was provided with a multilayer plastic protective case.

The novelty in the study presented in this paper is the fact

A first examination of data presented in the tables has shown that results are generally consistent with published tests done by other laboratories. According to our measurements, the cheek SAR values are higher than tilt SAR values, and 1-g SAR values are higher than $10-\mathrm{g}$ SAR values. Also, the $900-\mathrm{MHz}$ SAR values are higher than $1800-\mathrm{MHz}$ ones. These findings have theoretical support and are in good agreement with most of the other results revealed in literature.

We have noticed an anomaly in our measurements for Nokia mobile phone (the orange marked field in Table 3) for GSM1800 on the left side of the phantom in tilt position: the SAR values were higher than those determined on the right side of the phantom, for the same position. This anomaly was due to the position of the phone in the clamping device. After we have carefully verified and repositioning the phone, the measurement was reset and correct data has been taken. The situation illustrates the occasional abnormal, inexplicable values that have been also reported in comparative studies between laboratories.

Similar studies developed in other laboratories (so called intercomparison measurements) support the expectation that for a given mobile phone, frequency and position, SAR measurements on the left and right ear positions of the SAM phantom should be very close in value. When they are not, we should check for a user error in phone placement or in data recording.

As a first recommendation, the tilt position should be preferred by any user.

The designers of HUAWEI P20 Pro placed the antenna at the bottom, to be farther away from the user's brain. This is a major advantage versus the older mobile phones, their antenna being positioned on the top of the device.

As in principle expected, the lowest values of SAR had been recorded when the phone was provided with protective case.

Regarding the use of a protective casing, it is important that the material from which is made to be good absorbent; the protective cases having conducting insertions should be avoided, mainly due to unexpected and uncontrolled reflections. Future studies on this topic should involve testing different types of cases, to comparatively track their impact on SAR values.

Contrariwise, the transmission efficiency of the phone provided with protective case decreases. As a shortcoming, in this situation the battery will run out faster because it will try to give more power to ensure coverage, respectively a better signal reception. Anyway, in real, daily environment, the SAR values might vary depending on propagation conditions.

A general conclusion could be the following: a combination of factors such as the positioning of the antenna, the size of the device, the relative position to the human head, equipping the phone with a protective case, can lead to lower SAR values, regardless the type of the mobile terminal. 
The dosimetry evaluation here presented demonstrates that SAR maxim values for $10 \mathrm{~g}$ of tissue determined for all three mobile phones are smaller than the ICNIRP limit: $2 \mathrm{~W} / \mathrm{kg}$ (head region).

Carrying out rigorous measurements, in the case of most diverse exposure scenarios, with the correct processing of the results is an important resource to remove exaggerated fears, but also to develop recommendations and guidelines for effective reduction of human exposure to environmental electromagnetic fields. In this frame of such universal interest, any rigorous technical-scientific approach should be definitely welcomed.

\section{ACKNOWLEDGMENT}

This paper could be developed due to the collaboration agreement settled between "Gheorghe Asachi" Technical University of Iasi, Faculty of Electrical Engineering and LICETER accredited laboratory of ANCOM- Romania.

\section{REFERENCES}

[1] International Commission on Non-Ionizing Radiation Protection, Guidelines for Limiting Exposure to Time-Varying Electric, Magnetic, and Electromagnetic Fields (up to $300 \mathrm{GHz}$ ), Health Physics, vol. 74, 2020, pp. 494-521.

[2] IEEE, IEEE Standard for Safety Levels with Respect to Human Exposure to Radio Frequency Electromagnetic Fields, $3 \mathrm{kHz}$ to $300 \mathrm{GHz}, \mathrm{C} 95 . i 2005$. New York: Institute of Electrical and Electronics Engineers, 2005. Online [Accessed 20 June 2021] https://emfguide.itu.int/pdfs/C95.1-2005.pdf

[3] European Committee for Electrotechnical Standardization (CENELEC) Prestandard ENV 50166-2, Human exposure to electromagnetic fields. High frequency $(10 \mathrm{kHz}$ to $300 \mathrm{GHz})$. Online [Accessed 20 June 2021] https://standards.globalspec.com/std/85205/ENV\%2050166-2

[4] ORDER nr. 1193 from 29 September 2006 for the approval of the Norms regarding the limitation of the general population exposure to electromagnetic fields from $0 \mathrm{~Hz}$ to $300 \mathrm{GHz}$. Online [Accessed 20 June 2021], [In Romanian]

https://www.ancom.ro/uploads/links files/Odinul 11932006 norme.pdf

[5] W. Kainz, A. Christ, T. Kellom, S. Seidman, N. Nikoloski, B. Beard, N. Kuster, Dosimetric comparison of the specific anthropomorphic mannequin (SAM) to 14 anatomical head models using a novel definition for the mobile phone positioning, Physics in Medicine and Biology 50(14), August 2005, pp. 34233445.

DOI: $10.1088 / 0031-9155 / 50 / 14 / 016$

[6] C. C. Gordon, T. Churchill, C. E. Clauser, B. Bradtmiller, J.T. Mc Conville, I. Tebbetts, R. A. Walker, 1988 Anthropometric Survey of U.S. Army Personnel: Methods and Summary Statistics. Technical Report NATICK/TR-89/044, U.S. Army Natick Research, Development and Engineering Center, Massachusetts: Natick, Sep. 1989. Online [Accessed 20 June 2021] http://mreed.umtri.umich.edu/mreed/downloads/anthro/ansur $\angle$ Gordon 1989.pdf

[7] S. Aqeel Abdulrazzaq, S. Jabir, J. Aziz, SAR Simulation in Human Head Exosed to RF Signals and Safety Precautions, IJCSET, September 2013, Vol 3, Issue 9, pp. 334-340. Online [Accessed 20 June 2021]
http://www.ijcset.net/docs/Volumes/volume3issue9/ijcset2013 030908.pdf

[8] Prabir Kumar Dutta, Pappu Vankata Yasoda Jayasree, Viriyala Satya Surya Narayana Srinivasa Baba, SAR reduction in the modelled human head for the mobile phone using different material shields, Hum. Cent. Comput. Inf. Sci. 6 (2016), art. 3. DOI: $\underline{10.1186 / \mathrm{s} 13673-016-0059-0}$

[9] M. R. Iqbal-Faruque, N. Aisyah-Husni, Md. Ikbal-Hossain, M. Tariqul-Islam, N. Misran, Effects of Mobile Phone Radiation onto Human Head with Variation of Holding Cheek and Tilt Positions, Journal of Applied Research and Technology, Volume 12, Issue 5, October 2014, pp. 871-876.

DOI: $10.1016 /$ S1665-6423(14)70593-0

[10] L. Belrhiti, F. Riouch, A. Tribak, J. Terhzaz, Investigation of Dosimetry in Four Human Head Models for Planar Monopole Antenna with a Coupling Feed for LTE/WWAN/WLAN Internal mobile phone, Journal of Microwave, Optoelectronics and Electromagnetic Applications, Vol. 16, No. 2, June 2017, DOI: $10.1590 / 2179-10742017 \mathrm{v} 16 \mathrm{i} 2748$

[11] Ovidiu Bejenaru, Catalin Lazarescu, Alexandru Salceanu, Valeriu David, Study Upon Specific Absorption Rate Values for Different Generations of Mobile Phones by Using a SATIMO-COMOSAR Evaluation Dosimetry System, 12th International Conference and Exhibition on electromechanical and energy systems, Sielmen 2019, Chisinău, Moldova, 10-11 October 2019, pp. 1-5. DOI: $10.1109 /$ SIELMEN.2019.8905798

[12] M. A. Stuchlyans, S. Stuchly, Experimental radio and microwave dosimetry, in Polk Ch., Postow E., Handbook of Biological Effects of Electromagnetic Fields, (sec. edition), CRC Press, Boca Raton, N. Y., London, Washington DC, 1996, pp. 295-336.

[13] SAM Phantom on MVG website. Online [Accessed 20 June 2021] https://www.mvg-world.com/en/products/sar/saraccessories/sam-phantom

[14] EN 50361: Basic Standard for the Measurement of Specific Absorption Rate Related to Human Exposure to Electromagnetic Fields from Mobile Phones ( $300 \mathrm{MHz}-3 \mathrm{GHz}), 2001$. Online [Accessed 20 June 2021] https://standards.globalspec.com/std/532912/EN\%2050361

[15] IEEE standard 1528-2003: IEEE Recommended Practice for Determining the Peak Spatial-Average Specific Absorption Rate (SAR) in the Human Head from Wireles Communications Devices: Measurement Techniques, 19 December 2003, pp.1-120 DOI: $10.1109 /$ IEEESTD.2003.94414

[16] Industrial robots on Kuka website. Online [Accessed 20 June 2021]

https://www.kuka.com/en-de/products/robotsystems/industrial-robots

[17] OpenSAR V5 on on MVG website. Online [Accessed 20 June 2021]

https://www.mvg-world.com/en/products/field product family/sar-38/opensarv5

[18] ASRO, STANDARD SR EN 62209-1: Human exposure to radio frequency fields from hand-held and body-mounted wireless communication devices - Human models, instrumentation, and procedures -- Part 1: Procedure to determine the specific absorption rate (SAR) for hand-held devices used in close proximity to the ear (frequency range of $300 \mathrm{MHz}$ to $3 \mathrm{GHz}$ ), 2007. Online [Accessed 20 June 2021], [In Romanian] https://magazin.asro.ro/ro/standard/117718 